\title{
¿Entrenamos como bloqueamos? Estudio de caso aplicado a la superliga de voleibol masculina
}

Do we train as we block? Case study applied to the men's volleyball super league

\author{
E. Hernández-Hernández \\ Departamento de deporte e informática. Universidad Pablo de Olavide de Sevilla \\ ehernandez@upo.es
}

\begin{abstract}
Resumen
El objetivo del estudio fue revisar cómo se debe enseñar la técnica de desplazamiento en bloqueo, en función de lo que indican los manuales de entrenamiento; y posteriormente, comprobar las técnicas utilizadas por los jugadores centrales de la liga española de voleibol. Para ello, se realizó una revisión bibliográfica de los principales artículos y manuales de entrenamiento, sobre la técnica de ejecución del desplazamiento y bloqueo en voleibol. Para el estudio de los bloqueadores se utilizó una muestra compuesta por 275 acciones de bloqueo realizadas por los jugadores centrales (11 partido analizados), correspondientes a la primera vuelta de la liga 2016-17. Se utilizó un diseño descriptivo, observacional no participante. Se consideró como variable de medida la técnica de desplazamiento y el tipo de movimiento de brazos utilizados por los jugadores al bloquear. Los resultados muestran que la técnica más utilizada por los jugadores para desplazarse son los pasos cruzados; y la técnica del "chicken wing" para el movimiento de brazos. En las aplicaciones prácticas se realizan aportaciones de cara al entrenamiento de estos jugadores, con la finalidad de adaptar mejor los contenidos del entrenamiento a la forma real con la que se juega en competición.
\end{abstract}

Palabras claves: análisis del movimiento, técnicas de bloqueo, técnica de desplazamiento, jugador central.

\begin{abstract}
The aim of this study consisted in reviewing how the volleyball blocking technique should be taught, in comparison with the volleyball training handbooks. A review of the literatura dealing with the volleyball blocking technique and the footworrk pattern was led. The sample comprised 275 blocks performed by middle blockers in 11 sets (first phase 2016-17 Spanish regular League). A descriptive and observational design was implemented. The variables studied were the running step footwork pattern and the hands movement employed for blocking. The results shown that the "cross-over steps" and "chicken wing" were the most used techniques by the players. The practical applications' section presents a series of keypoints for improving the transfers between training and competition.
\end{abstract}

Keywords: motion analysis, volleyball blocking technique, running step footwork pattern, middle blocker.

Recibido: 15-oct-2019

Aceptado: 12-nov-2019

Publicado: 20-nov-2019

Como citar (apa): Hernández-Hernández, E. (2020). ¿Entrenamos como bloqueamos? Estudio de caso aplicado a la superliga de voleibol masculina. JUMP, (1), 9-16.

Como citar (vancouver): Hernández-Hernández, E. ¿Entrenamos como bloqueamos? Estudio de caso aplicado a la superliga de voleibol masculina. JUMP. 2020; (1), 9-16.
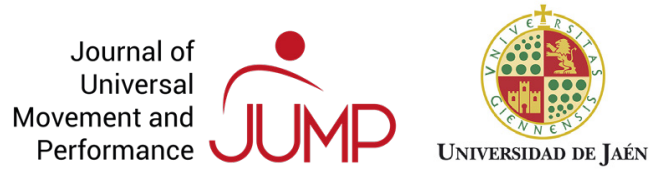


\section{Introducción}

El bloqueo es el primer elemento defensivo para frenar o contrarrestar las trayectorias del remate (Selinger \& Ackermann-Blount, 1986). Por ello, el bloqueo constituye el eje del sistema defensivo, y el punto de orientación para la defensa en campo. El bloqueo sólo puede ser realizado por los jugadores delanteros, y es el único elemento del juego que permite la acción simultánea de uno, dos o tres jugadores (FIVB, 2017). Éstos pueden sobrepasar la red en el plano vertical y horizontal tanto como quieran, pero no pueden tocar el balón antes de la terminación del ataque del equipo contrario (antes de que el balón sea colocado por el otro equipo). Con la aparición del bloqueo se inicia la concepción del voleibol moderno, y comienza a adquirir importancia la antropometría de los jugadores. El bloqueo es una de las habilidades más difíciles de dominar, ya que incorpora la capacidad atlética con la toma de decisiones (Hernández-Hernández et al., 2011). La demanda exigida a los bloqueadores ha aumentado porque el ataque se ha vuelto más explosivo y las combinaciones ofensivas se están jugando a velocidades más rápidas (Coleman y Neville, 1990). Los bloqueadores intentan contrarrestar estos cambios mediante capacidades como la anticipación y la rapidez en la toma de decisiones, llevadas a la práctica de forma coordinada con la ejecución de las técnicas más eficaces de desplazamiento (Neves, Johnson, Myrer y Seeley, 2011).

Durante un partido de voleibol, el jugador encargado de realizar el bloqueo por el centro (denominado como central) asiste a más bloqueos que los jugadores que lo realizan en el lateral de la red, ya que comparte la responsabilidad de asistir tanto al ataque que se realiza frente a él (zona $3)$, como los que se realizan en ambos laterales (zona 2 y 4), lo que implica recorrer más espacio (Millán-Sánchez, Morante, \& Ureña, 2018) y realizar más saltos (Manzanares, Ortega y Palao, 2015). Cuando se realiza una revisión bibliográfica de la técnica de desplazamiento más utilizada por a.

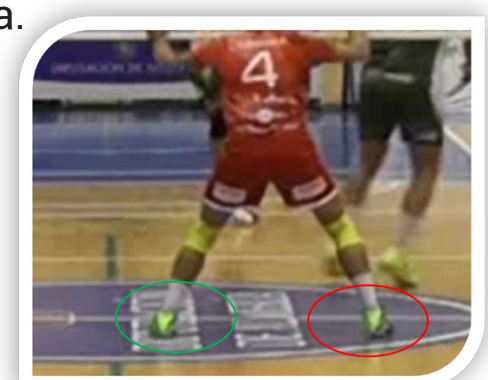

b.

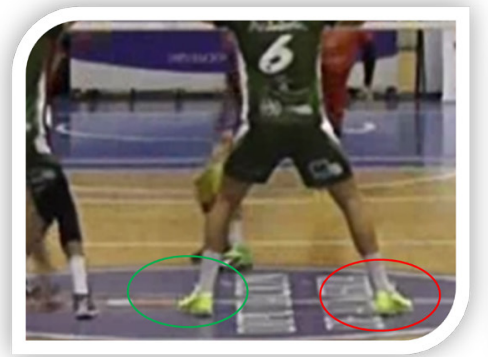

C.

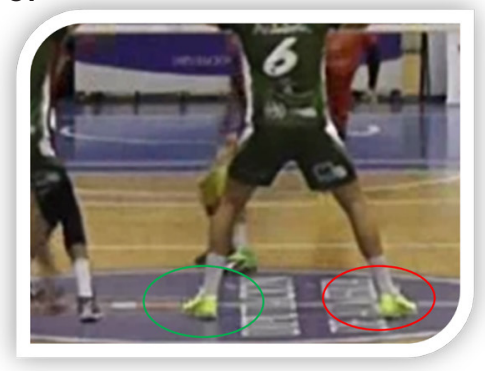

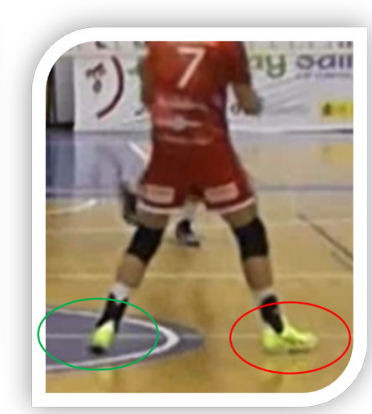
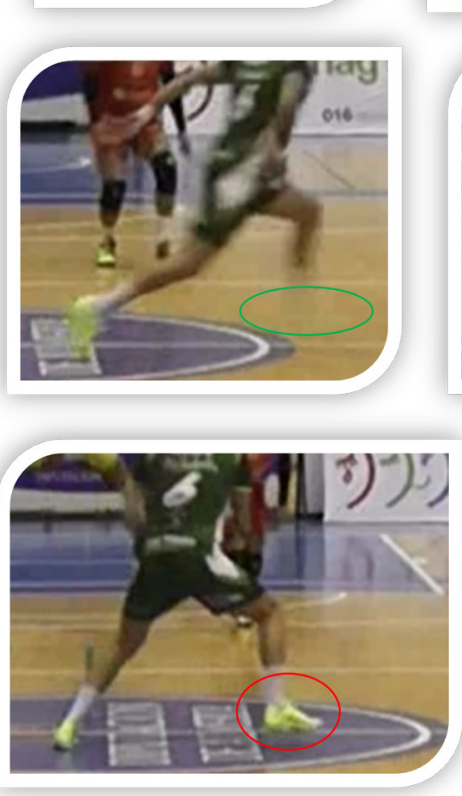

Figura 1. Imagen de las tres principales técnicas de desplazamiento más utilizadas por los jugadores centrales de voleibol. 
estos jugadores, lo que se obtiene es que las tres técnicas principales de desplazamiento son (figura 1): a) pasos deslizantes, b) pasos cruzados, o c) la combinación de ambos (Hernández, 1992; Lobietti \& Merni, 2006).

Otro de los aspectos clave para la ejecución de un bloqueo es el movimiento realizado por los brazos. Un uso eficiente de los brazos permite al jugador aumentar la altura del salto, y mejorar el ángulo de penetración de las manos, lo que se traducirá en una forma más efectiva de defender la red (Buekers, 1991; Cox et al., 1982; Farokhmanesh and McGown, 1988). Siguiendo la revisión realizada por Neves et al. (2011), las principales técnicas de movimiento brazos durante la ejecución de un bloqueo son tres (figura 2): a) la técnica denominada como "tradicional", que requiere que el jugador mantenga sus manos sobre el nivel del hombro a lo largo de todo el movimiento de bloqueo hasta el salto; b) la denominada como "swing" o balanceo, donde los brazos son inicialmente girados hacia atrás y luego avanza con los codos completamente extendidos a lo largo de todo el movimiento; y c) la denominada como "chicken wing",en la que la oscilación del brazo superior es la misma que el bloqueo de "swing" excepto que los codos se flexionan un ángulo de 90 grados a lo largo de los movimientos de oscilación hacia atrás y adelante.

No obstante, cuandose observa un encuentro de voleibol de alto nivel, en ocasiones, da la impresión, de que la técnica utilizada por los centrales se modifica con respecto a lo expuesto anteriormente. Aunque los manuales de entrenamiento consultados parecen definir de forma clara que estas son las tres técnicas que se deben enseñar, es frecuente ver cómo los jugadores realizan una adaptación de la batida del remate, que convierten en una técnica de desplazamiento, que les permite adquirir mayor altura de vuelo. A este respecto, el trabajo de revisión realizado por Marques, N.K. (2019) indica que el bloqueo realizado en carrera (lo que se puede entender como esa adaptación de la batida de remate), es la técnica de desplazamiento más rápida, lo que podría explicar su uso por estos jugadores. De la misma forma, Neves et al. (2011) concluyen en su estudio que la técnica del "swing o balanceo", es la técnica con la que se consigue mayor altura y grado de penetración de los brazos durante el salto, y, por tanto, tendría que ser la más utilizada por los jugadores centrales junto con el desplazamiento en carrera.

De esta forma, es posible que, a la hora de entrenar el movimiento de desplazamiento en bloqueo, y sobre todo en categorías inferiores, se esté entrenando la técnica de desplazamiento de forma diferente a lo que luego el jugador aplicará en el juego, cuando éste sea tan rápido que necesite un desplazamiento que implique más velocidad y mayor altura de vuelo. Lo que nos plantea el siguiente problema de trabajo: si se parte de la idea de que las técnicas de desplazamiento que
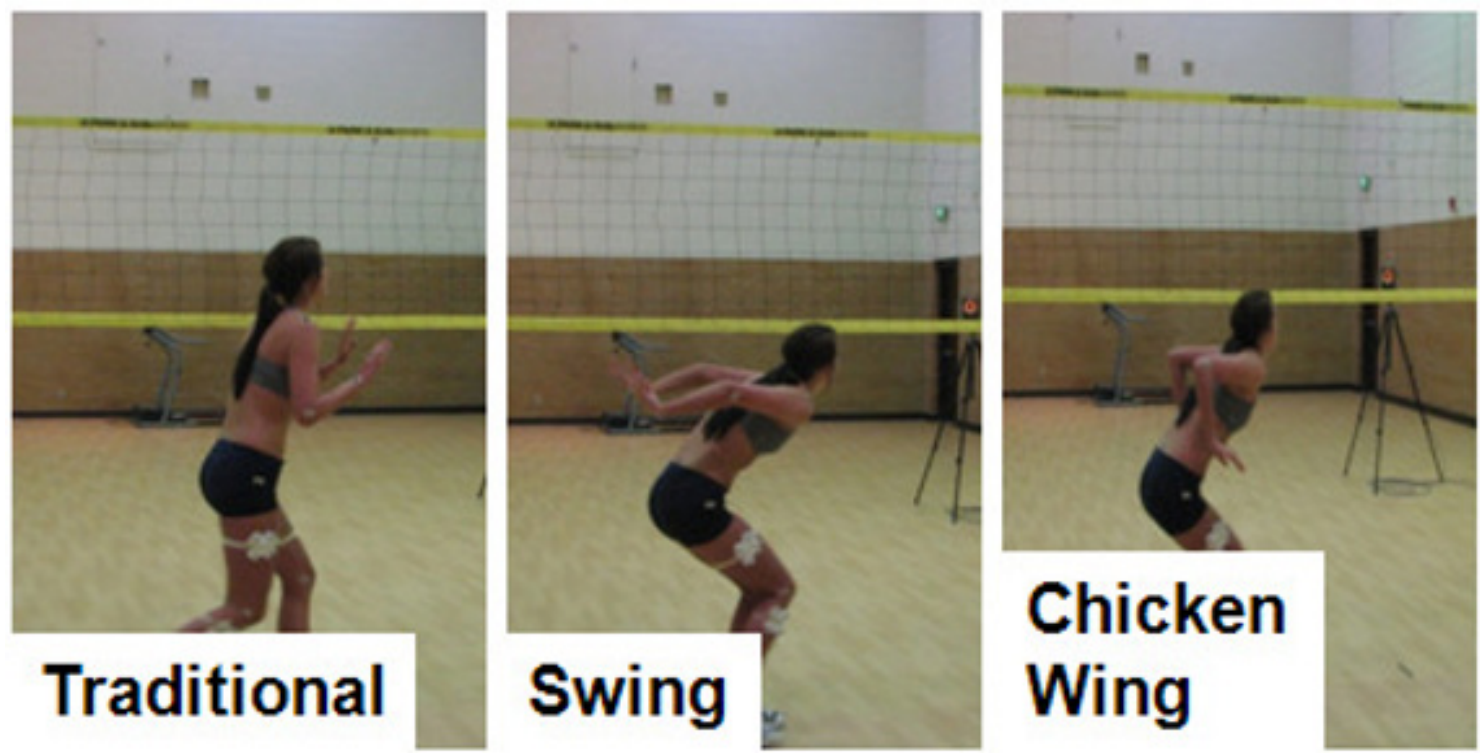

Figura 2. Ejemplo de las tres principales técnicas de movimiento de brazos utilizadas durante la realización del desplazamiento de bloqueo. Fuente: tomado de Neves et al. (2011). 
se enseña habitualmente son las que nos indican los manuales de entrenamiento, ¿se corresponden verdaderamente con la forma en la que se ejecuta durante la competición? De ahí que el propósito del trabajo que presentamos a continuación sea el de comprobar qué tipo de desplazamiento y movimiento de manos utilizan los centrales de la superliga de voleibol masculina, de cara a comprobar la forma en la que se ajustan a lo indicado por los manuales consultados.

\section{Método}

La muestra objeto de estudio estuvo compuesta por 275 acciones de bloqueo, correspondientes a un total de 12 sets analizados. Todas las acciones analizadas correspondieron a un set de los partidos disputados por el equipo de voleibol masculino Fundación Cajasol Juvasa contra el resto de los equipos participantes en la primera vuelta de la fase regular de la Superliga de voleibol masculina 2016-17. En total 11 partidos donde solo se han observado a los jugadores que realizan el bloqueo por el centro.

Para la revisión sistemática de la técnica de bloqueo se ha realizado una búsqueda de artículos y revistas mediante buscadores oficiales como Pubmed, Google Scholar, Athenea, EBSCOhost y SPORTDiscus, en estos dos últimos se encontraron 121 artículos relacionados con las palabras claves: "volleyball", "blocking", y "technique", de los cuales, 4 artículos estaban realmente relacionados con la temática de éste estudio. Además, se han utilizado otros recursos como libros y manuales de entrenadores a nivel autonómico y nacional.

Para el estudio observacional de los bloques, se ha utilizado un diseño descriptivo, observacional no participante (Anguera, 1991). Se consideró como variable de medida la técnica utilizada para bloquear por los jugadores centrales de la liga española. Para su observación, la técnica se ha divido en las siguientes categorías:

- Inferior derecho: esta variable analiza qué tipo de desplazamiento de los miembros inferiores ha utilizado el jugador cuando se desplazaba hacia la derecha y se ha valorado anotando a qué factor corresponde la jugada observada (1 = Lateral, 2 = Cruzado, 3=en carrera).

- Superior derecha: en esta variable se muestra el desplazamiento de los brazos que el jugador ha utilizado en cada acción hacia el lado derecho y se ha valorado anotando a qué factor corresponde la jugada observada ( $1=$ Tradicional, 2 = Swing, 3 = Chicken wings).

- Inferior izquierdo: esta variable indica el desplazamiento de los miembros inferiores ha utilizado el jugador cuando se desplazaba hacia la izquierda y se ha valorado anotando a qué factor corresponde la jugada observada ( 1 = Lateral, 2 = Cruzado, 3=en carrera).

- Superior izquierda: en esta variable se muestra el desplazamiento de los brazos que el jugador ha utilizado en cada acción hacia el lado izquierda y se ha valorado anotando a qué factor corresponde la jugada observada (1 = Tradicional, 2 = Swing, 3 = Chicken wings).

- Sin desplazamiento: este ítem indica que el ataque fue realizado por zona 3 , sin desplazamiento, con un movimiento de brazos tradicional.

El instrumento de registro consistió en una hoja de observación formato Microsoft Excel. Para la grabación de los partidos se utilizó una Videocámara Canon LEGRIA HF R706, 3.28MP CMOS, Full HD. Se realizó un entrenamiento del observador, obteniendo valores de fiabilidad de 0.95 .

Tabla 1. Técnica utilizada por los centrales de la liga española para iniciar el movimiento de los pies durante el bloqueo.

\begin{tabular}{lcccl}
\hline & $\begin{array}{l}\text { Técnica } \\
\text { utilizada }\end{array}$ & $\mathrm{n}$ & $\%$ & \\
\hline Bloqueo hacia la derecha & Lateral & 34 & $33,7 \%$ & $\begin{array}{l}\text { Movimiento de los pies utilizado para iniciar el } \\
\text { bloqueo hacia el lado derecho. }\end{array}$ \\
\cline { 2 - 5 } & Cruzado & 67 & $66,3 \%$ & $\begin{array}{l}\text { Movimiento de los pies utilizado para iniciar el } \\
\text { bloqueo hacia el lado izquierdo. }\end{array}$ \\
\cline { 2 - 5 } $\begin{array}{l}\text { Bloqueo hacia la } \\
\text { izquierda }\end{array}$ & Lateral & 12 & $15 \%$ & $\begin{array}{l}\text { Movimiento de los pies utilizado para iniciar el } \\
\text { bloqueo hacia el lado derecho. }\end{array}$ \\
\cline { 2 - 5 } & Cruzado & 68 & $85 \%$ & $\begin{array}{l}\text { Movimiento de los pies utilizado para iniciar el } \\
\text { bloqueo hacia el lado izquierdo. }\end{array}$ \\
\hline
\end{tabular}

Fuente: elaboración propia. 
Se ha realizado un análisis descriptivo de los datos, empleando porcentajes y frecuencias, a través del programa SPSS. 21.0.

\section{Resultados Y discusión}

Tras el análisis de los datos, se han obtenido un total de 275 jugadas en las que los bloqueadores realizaron una acción de bloqueo. De ellas, en 101 ocasiones el desplazamiento en bloqueo se realiza hacia el lado derecho de la red; en 80 el bloqueo se desplaza hacia el lado izquierdo de la red, y en 95 ocasiones el jugador salta desde el mismo centro, sin tener necesidad de realizar desplazamiento alguno (debido a que el ataque contrario se realiza por el centro de la red, en frente del jugador que está preparado para el bloqueo). Al igual que en los trabajos realizados por Afonso y Mesquita (2011), los resultados indican un predominio de los bloqueos hacia el lado derecho de la red (zona 4 del equipo contrario) en comparación con las otras dos zonas (centro y zona 2). Además, también refuerzan lo consultado en la bibliografía, donde la mayoría de los autores proponen la técnica del paso cruzado como la mejor opción porque permite recorrer una distancia mayor, lo cual es necesario cuando el jugador se desplaza desde el centro a bloquear en los laterales de la red (Hernández, 1992; Lobieti \& Merni, 2006; Ureña \& González, 2006; Selinger \& Ackermann-Blount, 1986).
Respecto a los resultados de las acciones que se llevaron a cabo con un desplazamiento previo, en la tabla 1 y 2 se exponen los resultados en función de la técnica utilizada, tanto para el miembro inferior (movimiento de pies), como para el miembro superior (movimiento de los brazos). En la primera de ellas se puede observar como la técnica predominante para desplazarse en bloqueo es la de "pasos cruzados" para ambos lados de la red. No obstante, se puede ver cómo esta diferencia es mayor cuando el bloqueo se realiza hacia el lado izquierdo de la red. Esto podría tener su explicación en el hecho de que se realicen más ataques por la zona 4 de la red, lo que puede influir sobre el jugador para que esté más predispuesto a iniciar el desplazamiento hacia la derecha. De esta forma, cuando el ataque se produce por el lado opuesto (zona 2), el jugador necesitará utilizar una técnica que implique llegar antes y en menos tiempo, y justifique el utilizar para ello la técnica de pasos cruzados (Buekers, 1991; Cox et al., 1982; Farokhmanesh \& McGown, 1988).

Los resultados correspondientes al movimiento que realizan los bloqueadores con los brazos vienen expuestos en la tabla 2. Se puede observar cómo mientras los resultados del bloqueo hacia el lado derecho están repartidos casi por igual entre las tres técnicas, para el lado izquierdo están más diferenciados. Para coger un mayor impulso a la hora de realizar el bloqueo, los jugadores

Tabla 2. Técnica utilizada por los centrales de la liga española para coger impulso con los brazos durante el bloqueo.

\begin{tabular}{|c|c|c|c|c|}
\hline & & $n$ & $\%$ & Criterio \\
\hline \multirow{3}{*}{ Bloqueo hacia la derecha } & Tradicional & 34 & $33,7 \%$ & $\begin{array}{l}\text { El jugador mantiene sus manos sobre el nivel } \\
\text { del hombro a lo largo de todo el movimiento de } \\
\text { bloqueo hasta el salto }\end{array}$ \\
\hline & Swing & 30 & $29,7 \%$ & $\begin{array}{l}\text { Los brazos son inicialmente girados hacia atrás } \\
\text { y luego avanza con los codos completamente } \\
\text { extendidos a lo largo de todo el movimiento de } \\
\text { bloqueo. }\end{array}$ \\
\hline & Chicken wings & 37 & $36,6 \%$ & $\begin{array}{l}\text { Existe oscilación del brazo superior pero los } \\
\text { codos se flexionan un ángulo de } 90 \text { grados a lo } \\
\text { largo de los movimientos de oscilación hacia } \\
\text { atrás y adelante }\end{array}$ \\
\hline \multirow{3}{*}{ Bloqueo hacia la izquierda } & Tradicional & 11 & $13,8 \%$ & $\begin{array}{l}\text { El jugador mantiene sus manos sobre el nivel } \\
\text { del hombro a lo largo de todo el movimiento de } \\
\text { bloqueo hasta el salto }\end{array}$ \\
\hline & Swing & 31 & $38,8 \%$ & $\begin{array}{l}\text { Los brazos son inicialmente girados hacia atrás } \\
\text { y luego avanza con los codos completamente } \\
\text { extendidos a lo largo de todo el movimiento de } \\
\text { bloqueo. }\end{array}$ \\
\hline & Chicken wings & 38 & $47,5 \%$ & $\begin{array}{l}\text { Existe oscilación del brazo superior pero los } \\
\text { codos se flexionan un ángulo de } 90 \text { grados a lo } \\
\text { largo de los movimientos de oscilación hacia } \\
\text { atrás y adelante }\end{array}$ \\
\hline
\end{tabular}

Fuente: elaboración propia. 
utilizan mayoritariamente las técnicas del "Swing o balanceo" y la de "Chicken wings". La técnica tradicional se utiliza en muy pocos casos (casi siempre cuando el bloqueo tiene que desplazarse poco). Las dos primeras ayudan a que el jugador coja más impulso, y ganar una mayor altura de vuelo, aspecto muy importante para asegurarse un bloqueo eficaz. Estos resultados coinciden con los obtenidos por Neves et al, (2011), en su caso, en una muestra de jugadoras femeninas. En el caso español estudiado, de forma general, la técnica más utilizada por los centrales de la liga es la técnica del "Chicken wings". Según los resultados obtenidos por Neves et al, (2011), esta es la técnica más rápida para despegar del suelo, y llegar rápido con las manos a realizar el movimiento de penetración y sellado de red, en comparación con la técnica tradicional, lo que iría en consonancia con los resultados encontrados. A pesar de que la técnica de "swing" permite alcanzar los mayores niveles de altura y ángulo de penetración de las manos, solo una tercera parte de las veces que se bloquea se utiliza. Este hecho, junto con la ausencia de situaciones en las que los jugadores en bloqueo se han desplazado mediante la técnica de carrera (técnica de desplazamiento que iría asociada a la técnica de swing o balanceo, para los brazos), hace pensar que los jugadores españoles observados no siempre utilizan la técnica más eficiente, con la que se consigue una mayor velocidad de desplazamiento (Marques, 2019), y se alcanza más altura (Neves et al., 2011). Por el contrario, la elección de la técnica a nivel de desplazamiento sigue la tendencia marcada por los manuales de entrenamiento (aplicar pasos cruzados en distancias grandes), mientras que, a nivel de brazos, no siempre utilizan la técnica más eficiente, y difiere en función de si el bloqueo se realiza en un lado $u$ otro de la red.

Por último, se analizaron los resultados obtenidos en función del ranking de clasificación que realiza la Real Federación española de voleibol al finalizar la liga. Este ranking establecer una clasificación por puestos, de todos los centrales participantes en función de la estadística acumulada por el jugador a lo largo de la liga. Los resultados hallados no muestran una relación clara entre la técnica utilizada por el jugador y su puesto de clasificación. Esto quiere decir que no está claro que los mejores bloqueadores de la liga utilizasen una técnica y los peores otra, en función del lado de la red.

\section{Aplicaciones prácticas}

El trabajo presentado permite hacer las siguientes recomendaciones, de cara al entrenamiento de bloqueadores centrales:

\section{Entrenar como se juega, desde las etapas de formación}

Los jugadores centrales están implicados en la mayoría de los bloqueos que se realizan en los laterales de la red. Esto implica que tienen que dominar técnicas que les permitan reaccionar rápido, llegar pronto y saltar alto. Se recomienda incluir desde las etapas de formación estas técnicas avanzadas de desplazamiento, que les permita entrenar para adaptarsealos requerimientos temporales que luego establece la alta competición (ataques veloces, diversidad de combinaciones de ataque y poco tiempo para reaccionar). Al igual que en otras posiciones de juego, si al joven jugador central, entre sus competencias, se le va a pedir que bloquee rápido y de manera eficiente, se debería preparar para eso desde las etapas de formación, no esperar a que el jugador tenga la necesidad de aprenderlas porque no llega a realizar bien sus funciones. Como entrenadores, debemos anticipar a estos problemas de juego, y darle herramientas al jugador para poder afrontarlo con garantías. Es decir, aunque en los manuales de iniciación no vengan de forma explícita estas técnicas de bloqueo, debemos incluirlas desde que el jugador comience su especialización en la posición de bloqueador central.

\section{Muévete más rápido}

Es necesario que los centrales no solo entrenen la técnica de paso cruzado, sino que en cuanto sea posible, entrenen e incluyan en su repertorio la técnica de desplazamiento en carrera, que es similar a la batida que se realiza en el remate. Con esta técnica se desplazarán más rápido, y se adaptarán mejor al juego rápido. Su ejecución ya es utilizada por los jugadores de alto nivel, llevándola a la práctica junto con el movimiento de brazos que hemos denominado como "swing 
o balanceo". La coordinación de ambas técnicas (desplazamiento + movimiento de brazos) obtendrá como resultado, al igual que ocurre con la batida del remate, una mayor velocidad y altura de vuelo, lo cual es uno de los parámetros esenciales para la formación de un bloqueo eficaz. En este caso, durante su entrenamiento, de recomienda vigilar la orientación del cuerpo en la fase de vuelo, para conseguir ubicarse paralelo a la red, y en buenas condiciones para realizar un sellado de la red eficaz. Dominar esta técnica ayudará al jugador bloqueador a ganar tiempo y su efectividad en bloqueo.

\section{Pon el tiempo de tu lado}

Los jugadores que se especialicen en la posición de central, deberían ser capaces de dominar las dos técnicas de brazos que permiten ganar mayor altura de vuelo, y un buen sellado de la red: la técnica del "chicken wing" y la técnica de "swing o balanceo". De esta forma, si el jugador se encuentra en situación de déficit de tiempo podrá optar por la primera, mientras que, si posee algo más de tiempo, podrá aplicar la segunda. Dominar ambos movimientos de brazos, le permitirá escoger en función de las condiciones del juego, y que no sea el juego el que condicione su técnica. Esto llevado al juego se traduce en, ocasiones donde el central tiene que asistir a combinaciones de ataque, con un alto compromiso en la zona central y ataques relativamente rápidos de los jugadores de ala. En este caso, la técnica del "chicken wings" permitirá al central poder reaccionar rápido y ayudar a esos ataques en el lateral, llegando con un buen ángulo de penetración de los brazos para poder sellar la red. Como el compromiso es fuerte en el centro, necesitará aplicar una técnica que le permita despegar lo más rápido que pueda, para compensar la velocidad del juego.

En otros casos, donde las circunstancias del juego hacen que sea algo más claro las opciones de ataque por los laterales de la red (recepciones de menos eficacia, contrataques, ataques zagueros por zona 1...), dominar la técnica del "swing o balanceo", permitirá al bloqueador llegar con una buena altura de salto, que, a su vez, le permita penetrar más las manos, y realizar un bloqueo más agresivo, por llegar en mejores condiciones. mejorará la eficacia en bloqueo frente a ataques potentes, que necesitan ser contrarrestados con bloqueos ofensivos, que tapen una superficie mayor de la red. El uso de esta técnica permitirá al jugador llegar condiciones óptimas para hacer esto. De cara al entrenamiento de esta técnica, se debe focalizar el trabajo a la correcta orientación de caderas y brazos, durante la fase de vuelo, que permitan ubicarse al jugador de frente al brazo ejecutor del atacante, y no adoptar posiciones con posibles "huecos" o brazos separados de la red (que podrían dar lugar a acciones de blockout).

\section{Agradecimientos}

Agradecer al cuerpo técnico del equipo de voleibol Fundación Cajasol Juvasa, que nos hayan proporcionado el material audiovisual, y a Rocío Roldán el análisis de las imágenes.

\section{Referencias}

Afonso, J. \& Mesquita, I. (2011). Determinants of block cohesiveness and attack efficacy in high-level women's volleyball. European Journal of Sport Science, 11(1), 69-75.

Anguera, M.T. (1991). Manual de prácticas de observación (3a edición). Trillas: México.

Buekers, M.J. (1991). The time structure of the block in volleyball: a comparison of different step techniques. Research Quarterly for Exercise and Sport, 62, 232-235.

Coleman, J. \& Neville, B. (1990). Blocking. Colorado Springs, USVBA.

Cox, R.H., Noble, L. \& Johnson, R.E. (1982). Effectiveness of the slide and cross-over steps in volleyball blocking--a temporal analysis. Research Quarterly for Exercise and Sport, 53, 101107

Farokhmanesh, M. \& McGown, C. (1988). A comparison of blocking footwork patterns. Coaching Volleyball, 1, 20-22

FIVB (2017). Manual para entrenadores de nivel II. Lausanne: Fédération Internationale de Volleyball.

Hernández, L. (1992). Los Deportes Olímpicos: Voleibol. Madrid: Impresos Izquierdos.

Hernández-Hernández, E., Oña, A., Bilbao, A., Ureña, A. \& Bolaños, J. (2011). Efecto de la aplicación de un sistema automatizado de proyección de preíndices para la mejora de la capacidad de anticipación en jugadoras de voleibol. Revista de Psicología del deporte, 20(2), 551-572.

Lobietti, R. \& Merni, F. (2006). Blocking footwork techniques used by male and female volleyball players are different. Journal Human Movement Study, 51(5), 307-320

Manzanares, P., Ortega, E. \& Palao, J.M. (2015). Specifity of technical and tactical training. A case study in volleyball. European Journal of Human Movement, 34, 139-155. 
Marques-Junior, N.M. (2019). Investigaciones sobre la ejecución del bloqueo en voleibol. Revista peruana de ciencias de la actividad física y el deporte, 6(2), 766-774.

Millán-Sánchez, A., Morante, J.C. \& Ureña, A. (2018). The middle blocker in volleyball: A systematic review. Journal of Human Sport and Exercise, in press. https://doi.org/10.14198/jhse.2019.141.03.

Neves, T.J., Johnson, W.A., Myrer, J.W. \& Seeley, M.K. (2011). Comparison of the traditional, swing, and chicken wing volleyball blocking techniques in NCAA division I female athletes. Journal of Sports Science and Medicine, 10, 452-457.

Ureña, A. \& González, M. (Ed.). (2006). Manual Preparador de Voleibol, Nivel 2. Andalucía, Sevilla: Jiménez-Mena.

Selinger, A. \& Ackermann-Blount, J. (1986). Arie Selinger's Power Volleyball: The Complete Guide to the Sport by the Coach of the Silver-medal-winning US Women's Olympic Volleyball Team. New York: St. Martin's Press 